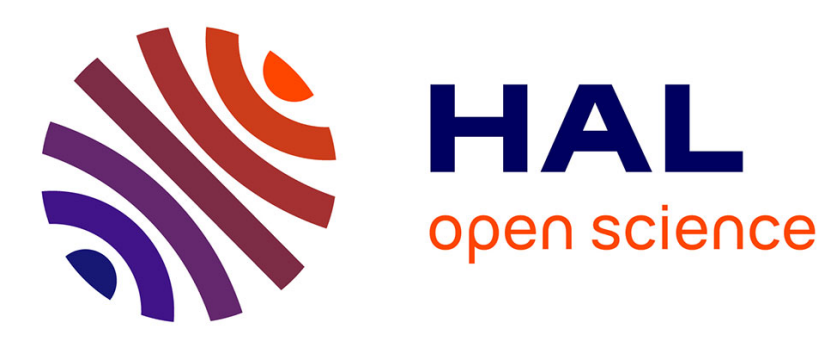

\title{
Reprise d'activité du cambium et xylogenèse chez l'Alisier torminal
}

\author{
Suzanne Lachaud, A. Mansouri
}

\section{To cite this version:}

Suzanne Lachaud, A. Mansouri. Reprise d'activité du cambium et xylogenèse chez l'Alisier torminal. Revue forestière française, 1993, 45 (3), pp.279-283. 10.4267/2042/26422 . hal-03443865

\section{HAL Id: hal-03443865 https://hal.science/hal-03443865}

Submitted on 23 Nov 2021

HAL is a multi-disciplinary open access archive for the deposit and dissemination of scientific research documents, whether they are published or not. The documents may come from teaching and research institutions in France or abroad, or from public or private research centers.
L'archive ouverte pluridisciplinaire HAL, est destinée au dépôt et à la diffusion de documents scientifiques de niveau recherche, publiés ou non, émanant des établissements d'enseignement et de recherche français ou étrangers, des laboratoires publics ou privés. 


\title{
REPRISE D'ACTIVITÉ DU CAMBIUM ET XYLOGENÈSE CHEZ L'ALISIER TORMINAL
}

\author{
Suzanne LACHAUD - A. MANSOURI
}

Le cambium, méristème essentiel pour la croissance en épaisseur des arbres, est formé de cellules qui se divisent activement au printemps et en été ; elles produisent les tissus conducteurs secondaires: phloème ou liber vers l'extérieur du tronc, xylème ou bois vers l'intérieur. L'étude du cambium, sorte d'usine à bois de l'arbre, et de son fonctionnement saisonnier ne semble pas encore avoir été faite chez l'Alisier torminal.

Nous aborderons ici quelques aspects de la réactivation cambiale chez l'Alisier, concernant d'une part la cytologie des cellules cambiales actives et du xylème en différenciation, d'autre part le mode de propagation de la reprise d'activité du cambium dans l'arbre entier au printemps.

\section{MATÉRIEL ET MÉTHODES}

\section{Matériel végétal}

Les Alisiers (Sorbus torminalis L. Crantz) mis à notre disposition sont des arbres jeunes et âgés, hauts respectivement d'environ $1,5 \mathrm{~m}$ et $15 \mathrm{~m}$; ils sont situés dans la forêt de La Roche (commune de La Roche-Posay, Vienne), sur un plateau argileux occupé par une chênaie.

Cytologie de la zone cambiale en activité

Une étude fine de l'ultrastructure des cellules cambiales et de leurs dérivées a été réalisée en microscopie électronique à transmission. Les échantillons de cambium utilisés proviennent de la tige de 1 an de jeunes alisiers récoltés le 19 mars (début de la réactivation) et le 30 mai (période d'intense activité cambiale).

On découpe des blocs de $1 \mathrm{~mm}^{3}$ environ contenant le cambium. La fixation des tissus, très délicate, est taite par le mélange glutaraldéhyde $3 \%+$ cacodylate $0,2 \mathrm{M}$ (soit 500 milliosmoles) à $\mathrm{pH} 7,2$ pendant 1 à 3 heures. Après trois lavages dans cacodylate $0,1 \mathrm{M}+$ saccharose $0,3 \mathrm{M}$, puis post-fixation au tétroxyde d'osmium à $1 \%+$ cacodylate $0,1 \mathrm{M}$ pendant 1 heure, les échantillons déshydratés sont inclus dans le Spurr ou l'Epon-Araldite. Les coupes transversales de cambium ont environ $0,06 \mu \mathrm{m}$ d'épaisseur.

\section{Propagation de la reprise de l'activité cambiale}

Chez trois alisiers âgés (A1, A2, A3), nous avons suivi l'évolution de l'état des cellules cambiales dans le temps (de mars à mai) et dans l'espace (tronc et branches), selon la méthode employée par Lachaud et Bonnemain (1981). À quatre dates 
successives: 19 mars, 8 avril, 26 avril, 30 mai, une carotte de $1 \mathrm{~cm}^{3}$ environ est prélevée à l'emporte-pièce dans la région cambiale tous les mètres le long du tronc et d'une branche; les tissus sont fixés dans l'éthanol à $60^{\circ}$.

Pour chaque échantillon, on détermine au microscope sur des coupes transversales de $20 \mu \mathrm{m}$ d'épaisseur le stade d'activité du cambium, selon les critères définis par Lachaud et Fazilleau (1987): cambium au repos (0), présence de quelques divisions $\left(0^{+}\right)$, mitoses nombreuses (R1), début de la xylogénèse (R2, R3), lignification du nouveau bois (L), bois initial différencié sur au moins une couche cellulaire (Bi).

\section{RÉSULTATS ET DISCUSSION}

\section{Cytologie du cambium en reprise d'activité}

Les cellules initiales fusiformes en reprise d'activité (figure 1A, ci-dessous) apparaissent bien vacuolisées et gonflées (réhydratées). Les parois tangentielles sont plus minces que les parois radiales.

À droite, dans une initiale fusiforme en fin de télophase, on repère la plaque cellulaire en élaboration (voir détail sur figure 1B, ci-dessous). Des profils de réticulum endoplasmique

Figure 1

REPRISE DE L'ACTIVITÉ CAMBIALE ET DE LA XYLOGENĖSE, EN MICROSCOPIE ÉLECTRONIQUE À TRANSMISSION.

A, B : 19 mars, coupe transversale du cambium de la tige de 1 an d'un jeune Alisier en cours de réactivation.

C : 30 mai, coupe transversale du xylème en différenciation et du bois différencié depuis l'année précédente, dans la tige de 1 an d'un jeune Alisier.

La longueur de la barre est indiquée en $\mu \mathrm{m}$.

$\sqrt{ }$ : limite du cerne ; $c$ : chloroplaste ; $L$ : inclusion lipidique ; $N$ : noyau ; pb : ponctuation bordée ; pc : plaque cellulaire ; pp : paroi primaire; pr : paroi radiale ; ps : paroi secondaire; pt : paroi tangentielle; PV : parenchyme vertical ; re : réticulum endoplasmique ; $T$ : trachéide ; $v$ : vacuole.
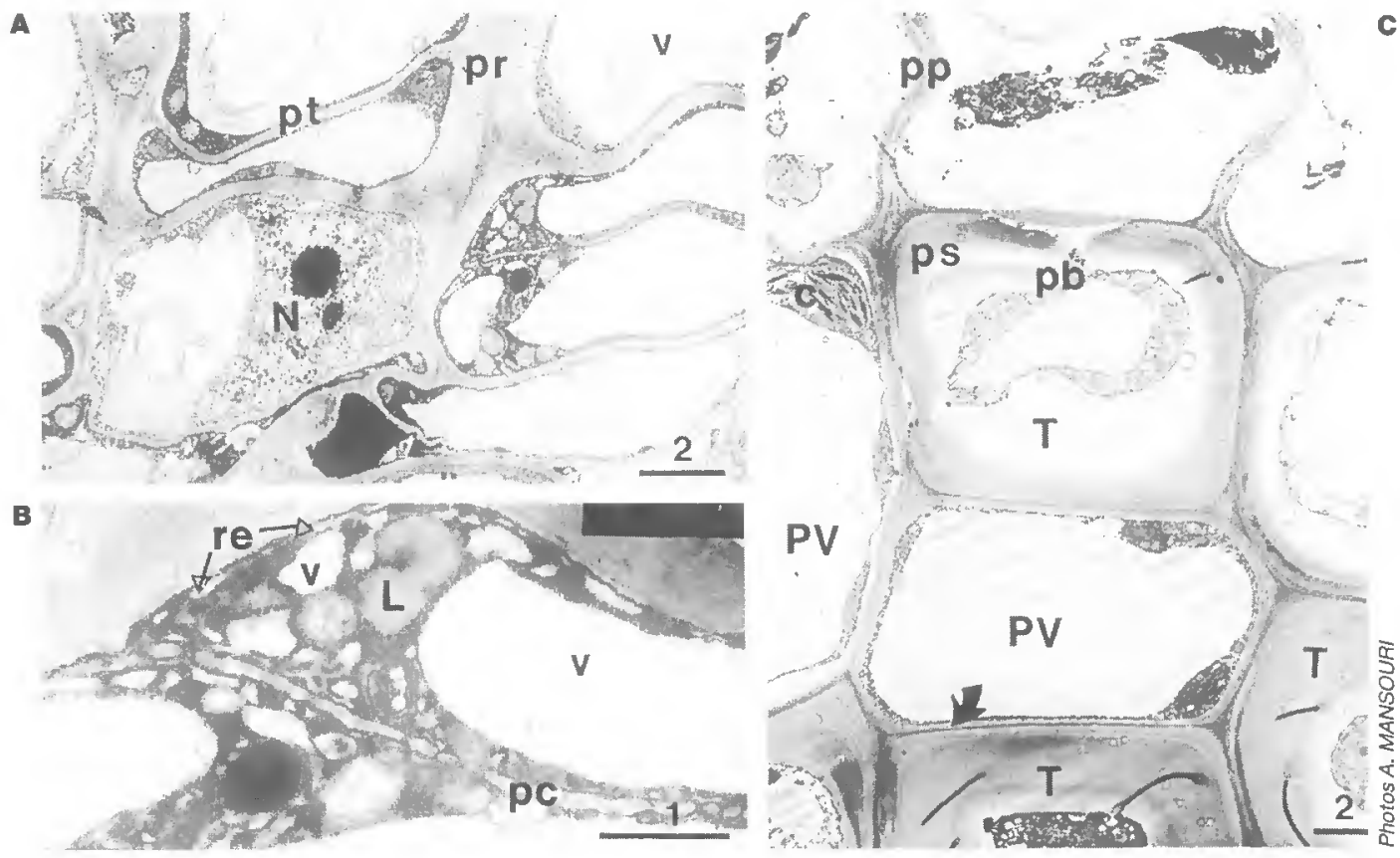

B

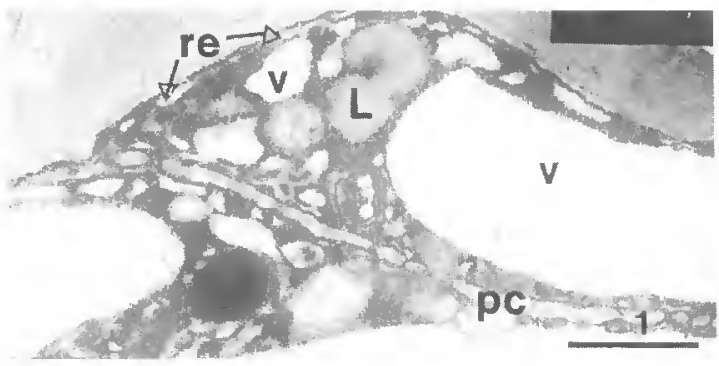


apparaissent sous forme de saccules allongés parallèles aux parois; ils se dilatent vers l'intérieur en petites vacuoles. Le cytoplasme contient notamment des globules lipidiques assez volumineux et des polyphénols (probablement des tannins).

L'ultrastructure des cellules cambiales de l'Alisier torminal en réactivation est comparable à celle que l'on observe chez d'autres espèces arborescentes (Catesson, 1974, 1990), notamment en ce qui concerne la construction des parois. La disposition du réticulum lors de la télophase, en saccules parallèles aux parois, semble caractériser les cellules cambiales en période d'activité (Faroqui et Robards, 1979). Les jonctions fréquentes que nous observons chez l'Alisier entre ce réticulum périphérique et des petites vacuoles, signalées aussi par Rao et Dave (1983) dans le cambium de Holoptelea en février, montrent la continuité du système endomembranaire, qui subit de profonds remaniements à cette époque (Catesson, 1990).

Les réserves lipidiques sont encore bien représentées dans les initiales fusiformes en mitose, alors que, chez d'autres espèces arborescentes, la dégradation des lipides cytoplasmiques est avancée à cette époque dans le cambium (Catesson, 1990). Ces lipides semblent relativement peu consommés au cours de l'évolution des dérivées xylémiennes; leur abondance a peut-être un rapport avec les propriétés " autolubrifiantes" du bois d'Alisier torminal.

\section{Cytologie du xylème en différenciation}

La figure 1C ( $p$. 280) montre quelques aspects de la xylogénèse dans les dérivées cambiales, notamment la construction des parois. Dans les cellules du haut, seule la paroi primaire a été déposée. On remarque que la lamelle moyenne, visible en gris clair au centre de la paroi radiale, n'est pas encore formée dans la paroi tangentielle qui est peu extensible, conformément aux observations des auteurs (Catesson, 1984).

Les cellules situées en dessous présentent un état de différenciation plus avancé. Celle du centre, dont le cytoplasme est altéré, présente déjà une épaisse paroi secondaire munie d'une ponctuation bordée: il s'agit probablement d'une trachéide (ce type de cellule sera défini dans l'article, p. 289). À gauche et en dessous, deux autres cellules ont une paroi secondaire relativement mince et une grande vacuole; dans leur cytoplasme, on repère des chloroplastes contenant de petits grains d'amidon: il s'agit probablement de cellules de parenchyme vertical.

La flèche noire marque la fin du cerne 1990. Les cellules situées en dessous correspondent donc à du xylème de $1 \mathrm{an}$, dans lequel on repère des trachéides à paroi très épaisse. La différenciation de ces cellules n'est pas terminée puisqu'elles contiennent encore du cytoplasme (dans le bois adulte, les trachéides paraissent vides comme les vaisseaux, leur cytoplasme ayant été lysé). II n'y a pas de vaisseaux dans le secteur observé.

\section{Progression de la réactivation cambiale et de la xylogenèse dans l'arbre entier}

La figure 2 (p. 282) résume les résultats obtenus. On constate d'abord que la reprise d'activité du cambium (stade $0^{+}, R 1$ ) est assez précoce: elle a déjà eu lieu le 19 mars pour les trois arbres étudiés; nous l'avons aussi constatée à cette époque chez les jeunes alisiers (figure $1 \mathrm{~A}$ et $1 \mathrm{~B}, \mathrm{p} .280$ ). Les bourgeons sont pourtant d'aspect hivernal (longueur inférieure à $1 \mathrm{~cm}$ ) le 19 mars chez les trois arbres et le 8 avril chez A2; lors de cette deuxième date de récolte, ils sont en gonflement chez $\mathrm{A} 1$ et débourrent chez $\mathrm{A} 3$.

Le cambium situé à l'extrémité de la branche présente toujours le stade le plus avancé (exemple: stade R2 le 19 mars chez A1; stade L le 8 avril chez A2). On observe parfois aussi ce stade à la même date à la base de la branche ou à un niveau quelconque du tronc (exemple: stade R1 le 19 mars chez $A 3$ à $10 \mathrm{~m}, 9 \mathrm{~m}$, et $8 \mathrm{~m}$ dans la branche, puis à $5 \mathrm{~m}$ dans cette même branche et à $3 \mathrm{~m}$ dans le tronc). Une exception semble se produire pour A2 le 19 mars: à 
l'extrẻmité de la branche, l'activité cambiale a moins évolué que dans sa partie basale (les niveaux $6 \mathrm{~m}$ et $7 \mathrm{~m}$ présentent du bois en lignification). Des blessures anciennes présentes à ces niveaux, à côté des prélèvements, peuvent expliquer ce dysfonctionnement local.

Figure 2

PROGRESSION DE LA RÉACTIVATION CAMBIALE ET DE LA XYLOGENĖSE DANS LE TRDNC ET LES BRANCHES DE TROIS ALISIERS ÂGÉS (NUMÉROTĖS 1, 2, 3), DU 19 MARS AU 30 MAI 1991.

$0,0^{+}, R 1, R 2, R 3, L, B i$ : étapes successives de la rẻactivation cambiale et de la xylogenèse Le trait noir appuyé représente la limite entre le tronc et la branche lors de chaque récolte.
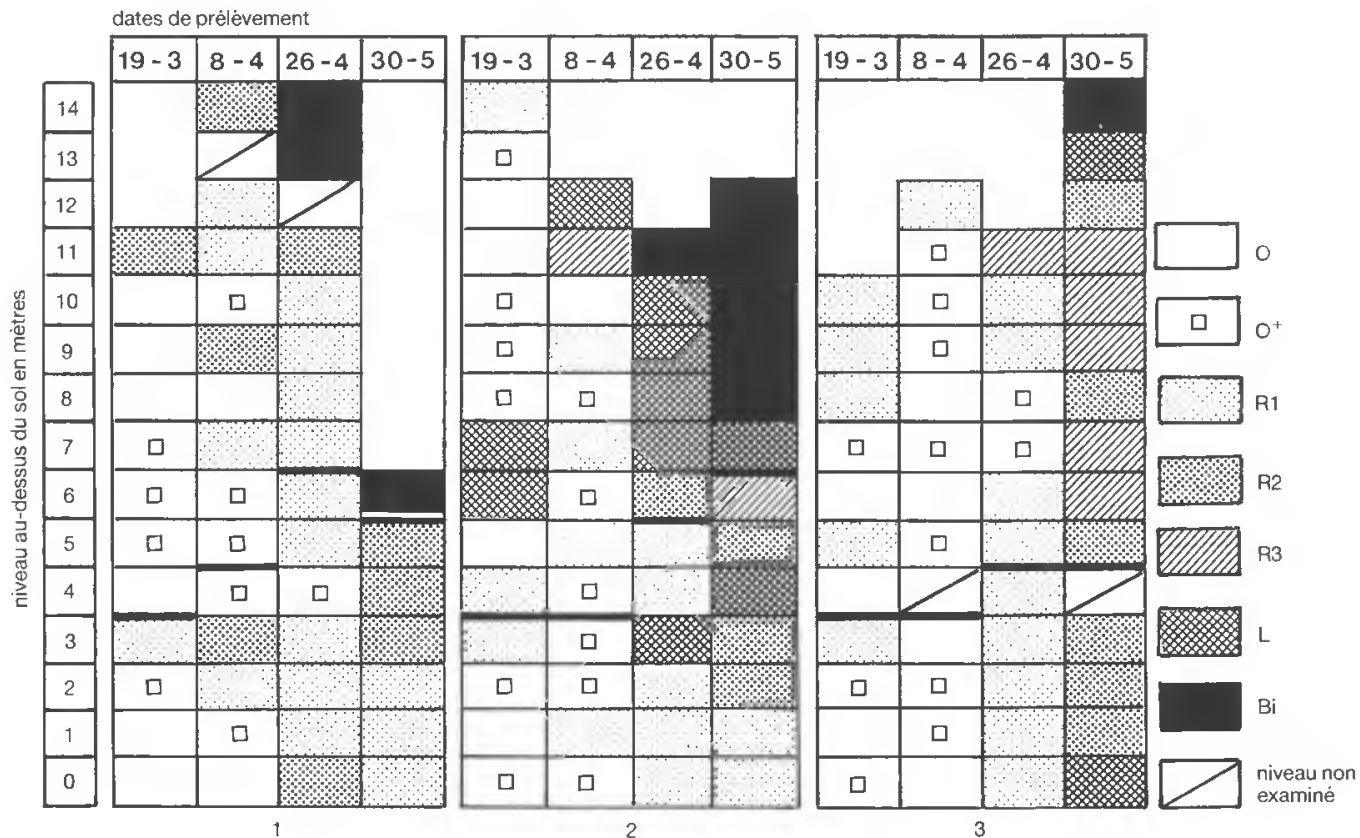

À l'extrémitè des branches, les stades observés présentent à chaque date une progression basipète sur 1 à $3 \mathrm{~m}$ (exemple : chez $\mathrm{A} 2$ : R1, $0^{+}, 0$ le 19 mars ; L, R3, 0 le 8 avril, etc...). A la base des branches et dans le tronc, les stades observés sont disposés dans le désordre et n'indiquent aucune propagation de la réactivation et de la xylogenèse dans l'espace.

Le début de la xylogénèse ( $R 2$, R3) est également assez précoce, on l'observe à l'apex le 19 mars chez A1, le 8 avril chez A2, soit avant le débourrement de ces deux arbres. Le stade le plus différencié (Bi) apparaît d'abord à l'extrémité des branches chez les trois arbres. Sa présence dans les troncs n'a pas encore été observée le 30 mai, l'évolution des dérivées cambiales etant plus lente dans les troncs que dans les rameaux.

La progression basipète de la réactivation cambiale dans l'extrémité des branches, ainsi que son apparition simultanée à divers niveaux du tronc a déjà été observée chez d'autres arbres à pores diffus âgés comme le Hêtre (Lachaud et Bonnemain, 1981). Toutefois, le comportement de l'Alisier âgé se rapproche davantage de celui du Bouleau ou de l'Érable champêtre (arbres à pores diffus), chez lesquels la base des branches est capable d'une réactivation gènéralisée du cambium en même temps que le tronc (Boutin, 1985). On sait que le tronc de l'Alisier torminal, 
relativement court ( 3 à $4 \mathrm{~m}$ ), se ramifie souvent en un groupe de grosses branches-maîtresses plus ou moins orthotropes. Ces grosses branches présenteraient donc une physiologie voisine de celle du tronc, impliquant une relative autonomie de leur cambium vis-à-vis des bourgeons.

En résumé, la reprise des divisions cellulaires est précoce dans le cambium de l'Alisier torminal: elle a lieu en Poitou à la mi-mars, avant le gonflement des bourgeons; les cellules en mitose contiennent alors des réserves lipidiques non altérées. Dans le nouveau bois en différenciation, les futures trachéides se caractérisent par l'épaississement rapide de leur paroi secondaire. Dans le tronc et les grosses branches-maîtresses, la réactivation cambiale se produit simultanément à divers niveaux.

\section{Remerciements}

Nous remercions particulièrement Melle A.-M. Catesson (Laboratoire des Biomembranes et Surtaces cellulaires végétales, ENS - Paris), Mme C. Besse (Service universitaire de Microscopie électronique appliquèe à la Biologie, Poitiers) et M. Sauvé (CRPF du Poitou-Charentes) pour leurs conseils et l'aide qu'ils nous ont apportèe.

\section{BIBLIOGRAPHIE}

BOUTIN (B.). - Étude de la réactivation cambiale chez un arbre ayant un bois à zone poreuse (Castanea sativa) et deux au bois à pores diffus (Betula verrucosa, Acer campestre). - Canadian Journal of Botany, vol. 63, 1985, pp. 1335-1343.

CATESSON (A.-M.). - Cambial cells. In: Dynamic aspects of plant ultrastructures / A.W. Robards Ed. London: Mc Graw Hill, 1974.

CATESSON (A.-M.). - La Dynamique cambiale. - Ann. Sci. nat. bot., vol. 13, nº 6, 1984, pp. $23-43$.

CATESSON (A.-M.). - Cambial cytology and biochemistry. In : Radial growth of plants / M. Iqbal Ed. Research Studies Press, 1990.

FAROQUI (P.), ROBARDS (A.W.). - Seasonal changes in the ultrastructure of cambium of Fagus sylvatica L. Proc. Ind. Acad. Sci., 88B, vol. 2, $n^{\circ} 6,1979$, pp. 463-472.

LACHAUD (S.), BONNEMAIN (J.-L.). - Xylogénèse chez les Dicotylédones arborescentes. I. Modalités de la remise en activité du cambium et de la xylogénèse chez les Hêtres et les Chênes âgés. - Canadian Journal of Botany, vol. 59, n 7,1981 , pp. 1222-1228.

LACHAUD (S.), FAZILLEAU (C.). - Inertie et réactivation cambiale chez le Hêtre. - Bulletin de la Société botanique de France, vol. 134, Actualites botaniques, $\mathrm{n}^{\circ} 3-4,1987$, pp. 7-18.

RAO (K.S.). DAVE (Y.S.). - Ultrastructure of dormant cambium in Holoptelea integrifolia (Roxb.). - Flora, vol. 174, 1983, pp. 165-172. 